\title{
Editorial
}

\section{Recent Contributions to Fixed Point Theory and Its Applications}

\author{
Mohamed A. Khamsi, ${ }^{1,2}$ Hichem Ben-El-Mechaiekh, ${ }^{3}$ and Bernd Schroeder ${ }^{4}$ \\ ${ }^{1}$ Department of Mathematical Sciences, UTEP, El Paso, TX 79968, USA \\ ${ }^{2}$ Department of Mathematics and Statistics, King Fahd University of Petroleum \& Minerals, Dhahran 31261, Saudi Arabia \\ ${ }^{3}$ Department of Mathematics, Brock University, St. Catharines, ON, Canada L2S 3A1 \\ ${ }^{4}$ Program of Mathematics and Statistics, Louisiana Tech University, Ruston, LA 71272, USA \\ Correspondence should be addressed to Mohamed A. Khamsi; mohamed@utep.edu
}

Received 26 February 2014; Accepted 26 February 2014; Published 22 April 2014

Copyright (C) 2014 Mohamed A. Khamsi et al. This is an open access article distributed under the Creative Commons Attribution License, which permits unrestricted use, distribution, and reproduction in any medium, provided the original work is properly cited.

The flourishing field of fixed point theory started in the early days of topology with seminal contributions by Poincare, Lefschetz-Hopf, and Leray-Schauder at the turn of the 19th and early 20 th centuries. The theory vigorously developed into a dense and multifaceted body of principles, results, and methods from topology and analysis to algebra and geometry as well as discrete and computational mathematics. This interdisciplinary theory par excellence provides insight and powerful tools for the solvability aspects of central problems in many areas of current interest in mathematics where topological considerations play a crucial role. Indeed, existence for linear and nonlinear problems is commonly translated into fixed point problems; for example, the existence of solutions to elliptic partial differential equations, the existence of closed periodic orbits in dynamical systems, and more recently the existence of answer sets in logic programming.

The classical fixed point theorems of Banach and Brouwer marked the development of the two most prominent and complementary facets of the theory, namely, the metric fixed point theory and the topological fixed point theory. The metric theory encompasses results and methods that involve properties of an essentially isometric nature. It originates with the concept of Picard successive approximations for establishing existence and uniqueness of solutions to nonlinear initial value problems of the 1st order and goes back as far as Cauchy, Liouville, Lipschitz, Peano, Fredholm, and most particularly, Emile Picard. However, the Polish mathematician Stefan Banach is credited with placing the underlying ideas into an abstract framework suitable for broad applications well beyond the scope of elementary differential and integral equations. Metric fixed point theory for important classes of mapping gained respectability and prominence to become a vast field of specialization partly and not only because many results have constructive proofs, but also because it sheds a revealing light on the geometry of normed spaces, not to mention its many applications in industrial fields such as image processing engineering, physics, computer science, economics, and telecommunications.

A particular interest in fixed points for set-valued operators developed towards the mid-20th century with the celebrated extensions of the Brouwer and Lefschetz theorems by Kakutani and Eilenberg-Montgomery, respectively. The Banach contraction principle was later on extended to multivalued contractions by Nadler. The fixed point theory for multivalued maps found numerous applications in control theory, convex and nonsmooth optimization, differential inclusions, and economics. The theory is also used prominently in denotational semantics (e.g., to give meaning to recursive programs). In fact, it is still too early to truly estimate the importance and impact of set-valued fixed point theorems in mathematics in general as the theory is still growing and finding renewed outlets.

This special issue adds to the development of fixed point theory by focusing on most recent contributions. It includes works on nonexpansive mappings in Banach and metric spaces, multivalued mappings in Banach and metric spaces, monotone mappings in ordered spaces, multivalued mappings in ordered spaces, and applications to such nonmetric spaces as modular spaces, as well as applications to logic programming and directed graphs.

Mohamed A. Khamsi Hichem Ben-El-Mechaiekh Bernd Schroeder 


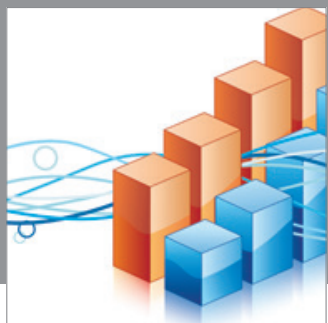

Advances in

Operations Research

mansans

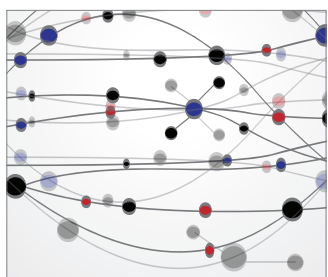

The Scientific World Journal
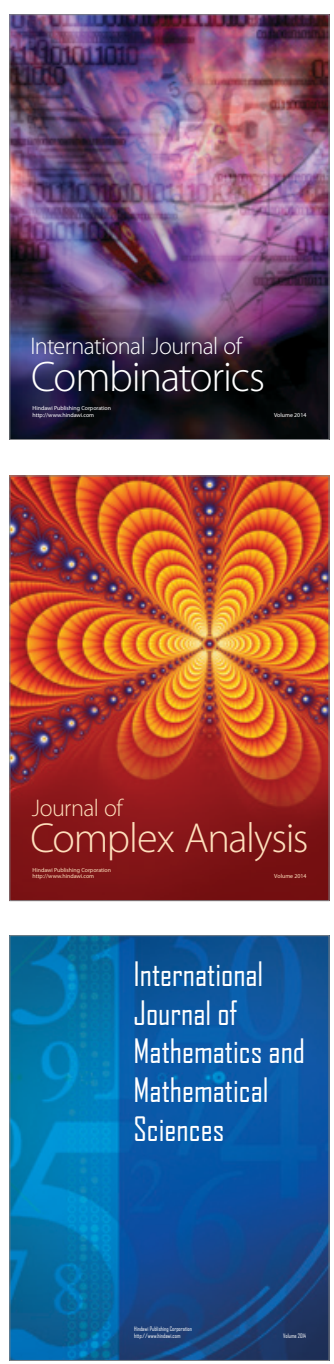
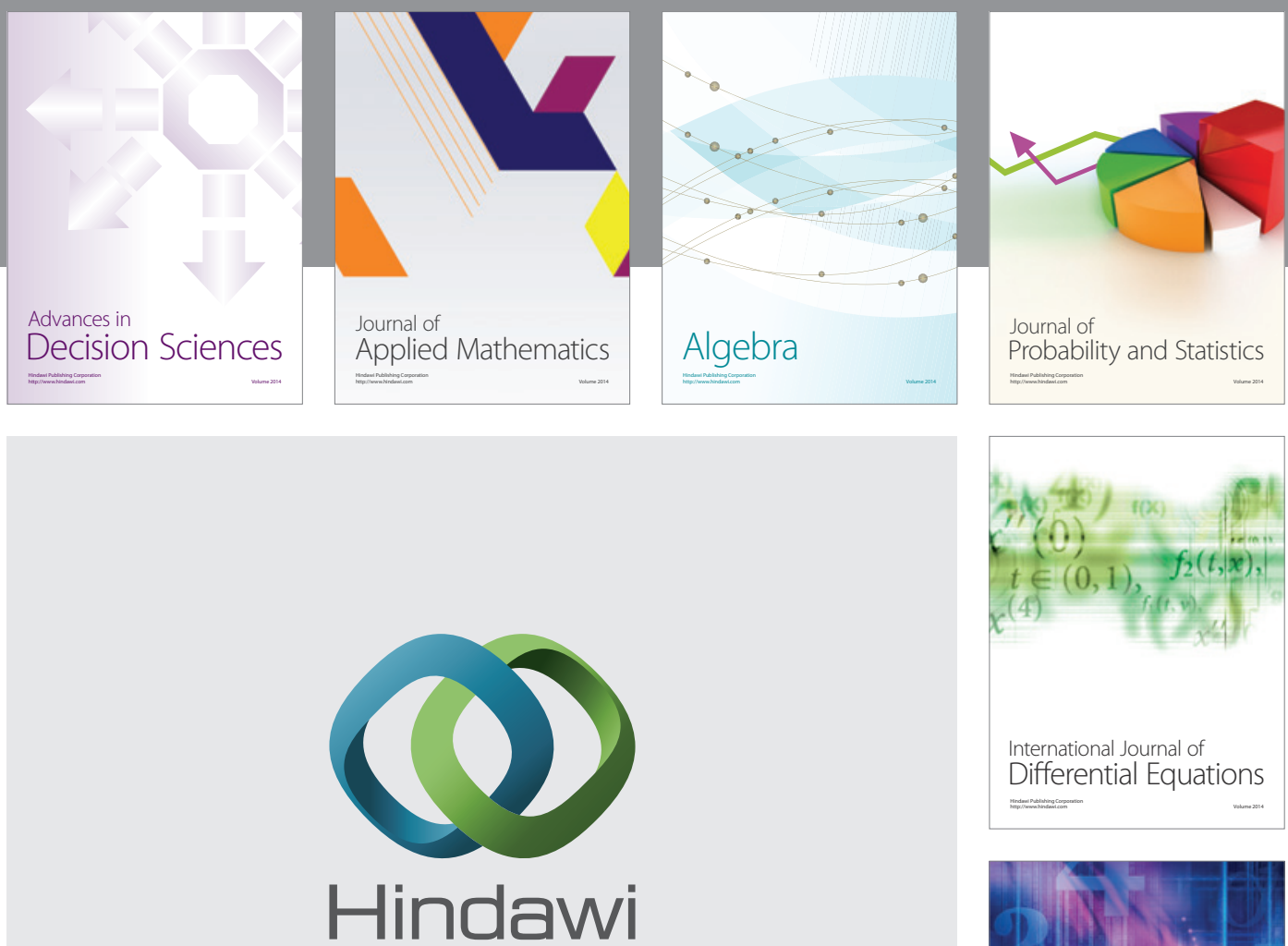

Submit your manuscripts at http://www.hindawi.com
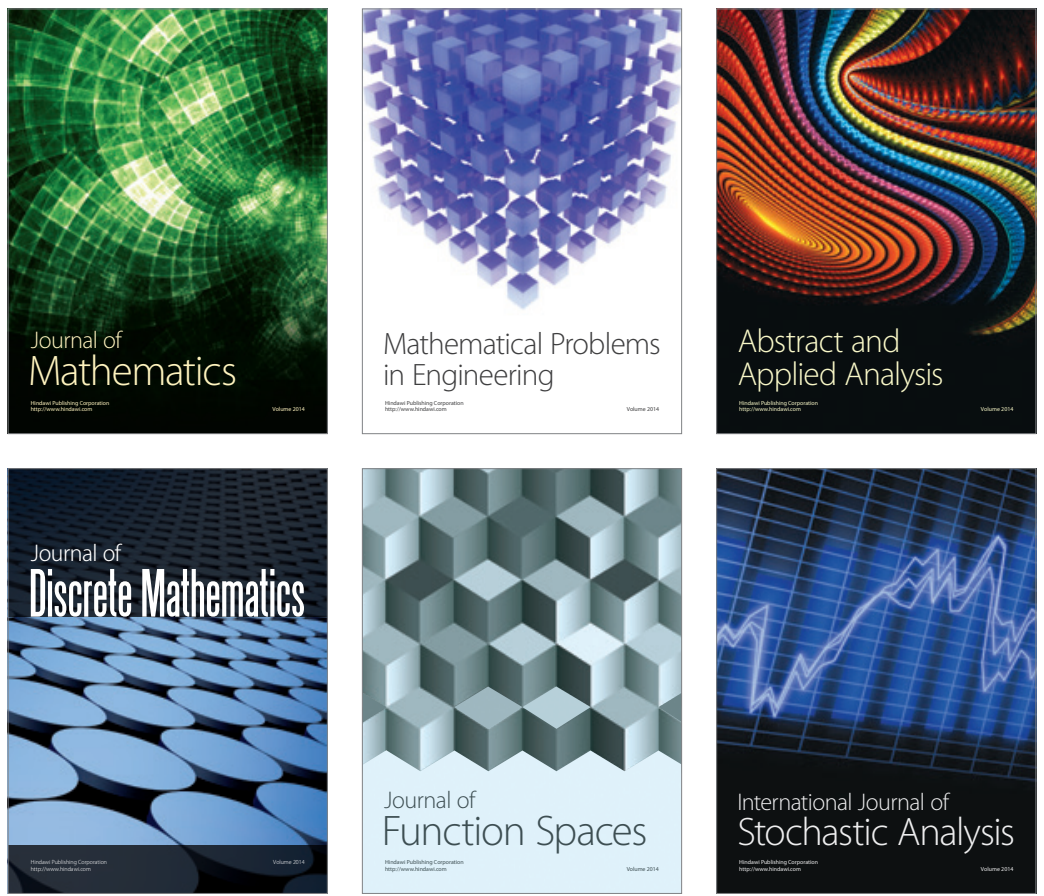

Journal of

Function Spaces

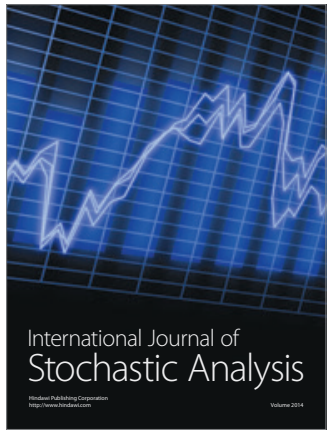

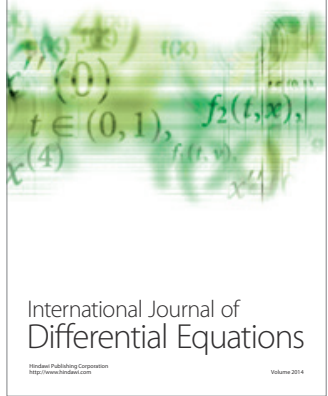
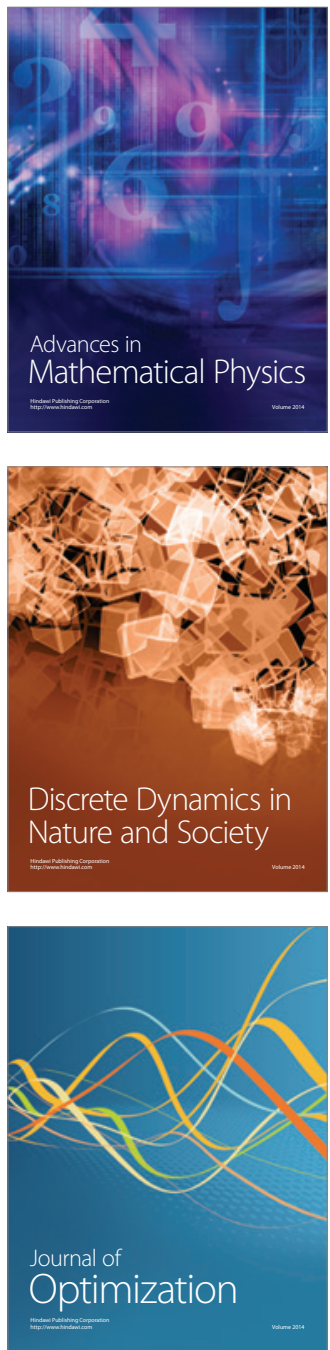